\title{
Adaptive Homodyne Measurement of Optical Phase
}

\author{
Michael A. Armen, ${ }^{*}$ John K. Au, John K. Stockton, Andrew C. Doherty, and Hideo Mabuchi \\ Norman Bridge Laboratory of Physics 12-33, California Institute of Technology, Pasadena, California 91125
}

(Received 1 April 2002; published 4 September 2002)

\begin{abstract}
We present an experimental demonstration of the power of feedback in quantum metrology, confirming the predicted [H. M. Wiseman, Phys. Rev. Lett. 75, 4587 (1995)] superior performance of an adaptive homodyne technique for single-shot measurement of optical phase. For measurements performed on weak coherent states with no prior knowledge of the signal phase, adaptive homodyne estimation approaches closer to the intrinsic quantum uncertainty than any previous technique. Our results underscore the importance of real-time feedback for reaching quantum limits in measurement and control.
\end{abstract}

DOI: $10.1103 /$ PhysRevLett.89.133602

Quantum mechanics complicates metrology in two complementary ways. First it forces us to accept the existence of intrinsic uncertainty in the value of observables such as the position, momentum, and phase of an oscillator. Such quantum uncertainty exists even when the state of the system being measured has been prepared in a technically flawless way. Even the vacuum state of a single-mode optical field, for example, exhibits "zeropoint" fluctuations in the amplitudes of its electric and magnetic components. The same is true of the optical coherent states representative of the output of an ideal laser. These uncertainties limit the sensitivity of ubiquitous measurement techniques such as laser interferometry. Squeezed states of light are challenging to produce but have reduced uncertainties that could be exploited for improved sensitivity in optical metrology [1]. To date, however, the achievable performance gains have not been sufficient to motivate their use in practical applications.

Quantum mechanics presents a second major obstacle to precision measurement by making it generally quite difficult to realize ideal measurement procedures, whose inherent inaccuracy is small enough to reveal the intrinsic uncertainty limits associated with quantum states. Let us refer to such ideal measurements as being uncertaintylimited (UL). Clearly, the implementation of UL measurement procedures is essential for any application that seeks to take advantage of exotic quantum states with reduced intrinsic uncertainties. In connection with squeezed states of light, measurements of optical quadrature amplitudes do constitute an important class of UL measurements that actually can be implemented in practice (via homodyne detection). But this is an unusual case as UL measurement schemes have not previously been demonstrated even for closely related observables such as optical phase, despite intense historical interest in their quantum properties [2,3]. This shortcoming is not merely one of achievable signal-to-noise ratio in realistic experiments - there has yet to be any demonstration of a measurement procedure that is capable even in principle
PACS numbers: 42.50.Dv, 03.65.Ta, 03.67.-a, 06.90.+v

of achieving UL estimation of true optical phase (as opposed to phase-quadrature amplitude), for coherent or any other pure states of an optical field.

In this Letter we present an experimental demonstration of the surprising efficacy of real-time feedback in the development of UL measurement procedures. We do this in the context of measuring the optical phases of weak pulses of light, following a theoretical proposal by Wiseman [4]. This metrological task can be motivated by a coherent optical communication scenario in which information is encoded in the phase of laser pulses that must travel long distances between the sender and receiver. In such a context the receiver is likely to be faced with decoding information carried by optical wave packets whose quantum states correspond to coherent states with low mean photon number (as a result of optical attenuation). If the sender is encoding information in an efficient manner, the variation of phase from one optical wave packet to the next should be uniformly distributed over the entire interval from zero to $2 \pi$. Hence, the receiver would ideally like to implement a single-shot UL measurement procedure for estimating the phase of each individual pulse [5]. The variance of such optimal phase estimates should be limited only by the intrinsic quantum uncertainty associated with optical coherent states of the given mean photon number.

There is no known experimental procedure to accomplish this goal exactly. Prior to Wiseman's proposal [4] it had been widely believed [6] that the best feasible strategy for single-shot phase decoding should be heterodyne detection, which for coherent signal states can in principle achieve a measurement variance that is only a factor of 2 greater than the intrinsic uncertainty limit [7]. In what follows we will thus consider heterodyne phase estimation as the benchmark we need to surpass. It is important to compare the current measurement scenario with what arises in applications such as the implementation of optical frequency standards (optical clocks), where optical phases must also be measured optimally but where there is a guarantee that the phases vary only over a range much 
less than $\pi / 2$. In such cases, as mentioned above, essentially UL measurement can be achieved by employing fixed-quadrature homodyne detection with a local oscillator whose phase is held at an offset of $\pi / 2$ radians from the expected signal phase. Real-time feedback can thus be seen as a key ingredient in formulating an UL scheme for measurement scenarios in which we have no prior knowledge of the signal phase - feedback enables protocols in which the local oscillator phase is adapted, in real-time, to the phase of each individual signal pulse [8]. As each optical pulse has some spatiotemporal extent, the measurement signal generated by the leading edge of a given pulse can be used to form a preliminary estimate of its phase, which is used promptly to adjust the local oscillator settings to be optimal for that pulse. (Note that this essentially amounts to the implementation of a quantumnoise limited phase-lock loop; the scheme relies on lock acquisition from the first one or two photons of signal in each pulse.) Detailed theoretical analyses of such schemes by Wiseman and co-workers have led to the striking realization that, despite extremely low signal-to-noise ratio in the feedback loop, adaptive homodyne measurement can be essentially UL for optical pulses with mean photon number of order 10 (or greater), and benefits of real-time adaptation should still be evident for mean photon numbers $\sim 1$. We turn now to our experimental test of these predictions.

The data plotted in Fig. 1(a) demonstrate the superiority of an adaptive homodyne measurement procedure ("adaptive") to the benchmark heterodyne measurement procedure ("heterodyne") for making single-shot estimates of optical phase. As described in detail below, we perform these measurements on optical pulses of $50 \mu \mathrm{s}$ duration derived from an intensity-stabilized cw laser. We have also plotted the theoretical prediction for the variance of ideal heterodyne measurement, both with (thin solid line) and without (dotted line) correction for a small amount of excess electronic noise in the balanced photocurrent. The excellent agreement between the heterodyne data and theory indicates that we have no excess phase noise (technically ideal preparation of coherent signal states) and validates electronic calibrations involved in our data analysis [9]. In the range of $\sim 10-300$ photons per pulse, the adaptive data lie well below the heterodyne curve that has been corrected for electronic noise (which also has a detrimental effect on the adaptive data), and a few of the data points lie significantly below the absolute limit. Quantitatively, for $\approx 50$ photons per pulse the adaptive data point sits 6.5 standard deviations below the absolute heterodyne limit (note logarithmic scale).

For intermediate values of the photon number the adaptive variances should ideally be even lower [10], but the performance of our experiment is limited by finite feedback bandwidth. For signals with large mean photon number, the adaptive scheme is inferior to heterodyne because of excess technical noise in the feedback loop. As the intrinsic phase uncertainty of coherent states becomes large for very low photon numbers, the relative differences among the expected variances for adaptive, heterodyne, and ideal estimation become small. Accordingly, we have been unable to beat the heterodyne limit for phase estimation variances with adaptive homodyne measurement for mean photon numbers $N \lesssim 8$. (Note that all theoretical curves in Fig. 1(a) correspond

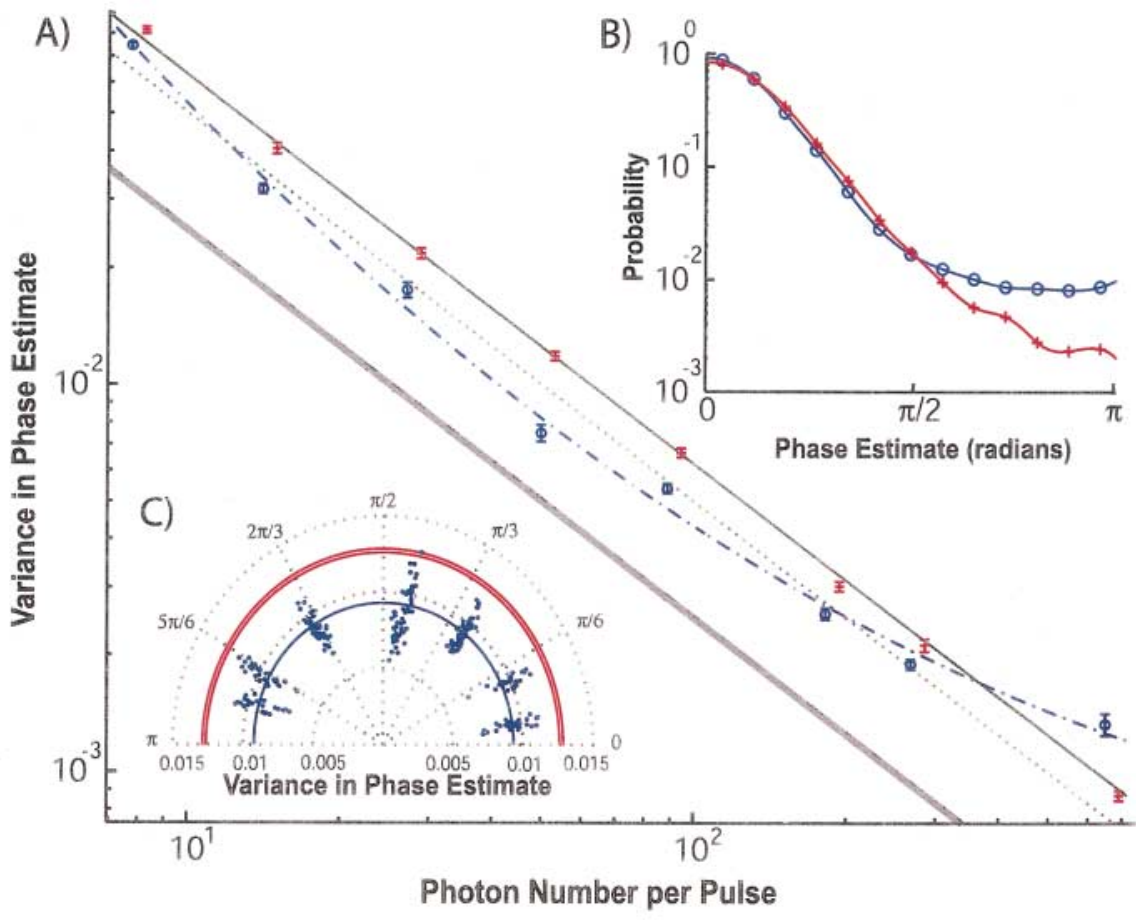

FIG. 1 (color). Experimental results from the adaptive and heterodyne measurements. (a) Adaptive (blue circles) and heterodyne (red crosses) phase estimate variance vs pulse photon number. The blue dash-dotted line is a second order curve through the adaptive data, to guide the eye. The thin lines are the theoretical curves for heterodyne detection with (solid) and without (dotted) corrections for detector electronic noise. The thick solid line denotes the fundamental quantum uncertainty limit, given our overall photodetection efficiency. (b) Phase-estimator distributions for adaptive (blue circles) and heterodyne (red crosses) measurements, for pulses with mean photon number $\approx 2.5$. (c) Polar plot showing the variance of adaptive phase estimates (blue dots) for different signal phases (mean photon number $\approx 50$ ). The solid blue line is a linear fit to the data. The double red lines indicate the $1 \sigma$ scatter for our heterodyne data, averaged over initial phase. 
to asymptotic expressions valid for large mean photon numbers; corrections are small for $N \gtrsim 10$.) However, we are able to show that the estimator distribution for adaptive homodyne remains narrower than that for heterodyne detection even for pulses with mean photon number down to $N \approx 0.8$. In Fig. 1 (b) we display the adaptive and heterodyne phase-estimator distributions for a signal size corresponding to $\approx 2.5$ photons. Note that we have plotted the distributions on a logarithmic scale, and that the mean phase has been subtracted off so that the distributions are centered at zero. The horizontal axis can thus be identified with estimation error. The adaptive phase distribution has a smaller "Gaussian width" than heterodyne but exhibits rather high tails, in qualitative agreement with predictions [11] based on quantum estimation theory. Theoretical analysis suggests that the high tails are a consequence of changes in the sign of the photocurrent caused by vacuum fluctuations of the optical field, which become comparable to the coherent optical signal for $N \sim 1$. The feedback algorithm responds to these photocurrent inversions by locking to incorrect phase values with an error that tends towards $\pi$.

Accurately assessing the performance of a single-shot measurement requires many repetitions of the measurement under controlled conditions. Figure 2(a) shows a schematic of our apparatus. Light from a single-mode cw Nd:YAG laser is first stripped of excess intensity noise by passage through a high-finesse Fabry-Perot cavity (not pictured) with ringdown time $\approx 16 \mu \mathrm{s}$; the transmitted beam is shot-noise limited above $\sim 50 \mathrm{kHz}$. This light enters the Mach-Zehnder interferometer (MZI) at beam splitter 1 (BS1), creating two beams with well-defined relative phase. The local oscillator (LO) is generated using an acousto-optic modulator (AOM) driven by an rf synthesizer RF1 at $84.6 \mathrm{MHz}$, yielding $\sim 230 \mu \mathrm{W}$ of frequency-shifted light. The signal beam corresponds to a frequency sideband created by an electro-optic modulator (EOM) driven by an rf synthesizer (RF2) that is phase locked to RF1. The power ( $5 \mathrm{fW}$ to $5 \mathrm{pW}$ ) and pulse length $(50 \mu \mathrm{s})$ of the signal beam are controlled by changing the amplitude of RF2 and by switching it on/off. A pair of photodetectors collect the light emerging from the two output ports of the final 50/50 beam splitter (BS2); the difference of their photocurrents [balanced photocurrent $I(t)]$ provides the basic signal used for either heterodyne or adaptive phase estimation [9]. At our typical LO power, the photodetectors supply $6 \mathrm{~dB}$ of shot noise (over electronic noise) in the difference photocurrent from $\sim 1 \mathrm{kHz}$ to $10 \mathrm{MHz}$. We perform adaptive homodyne measurement by feedback to the phase of RF2, which sets the (instantaneous) relative phase between signal and LO [12]. Our feedback bandwidth $\sim 1.5 \mathrm{MHz}$ is limited by the maximum slew rate of RF2. Real-time electronic signal processing for the feedback algorithm is performed by a field programmable gate array (FPGA) that can execute complex computations with very low delay

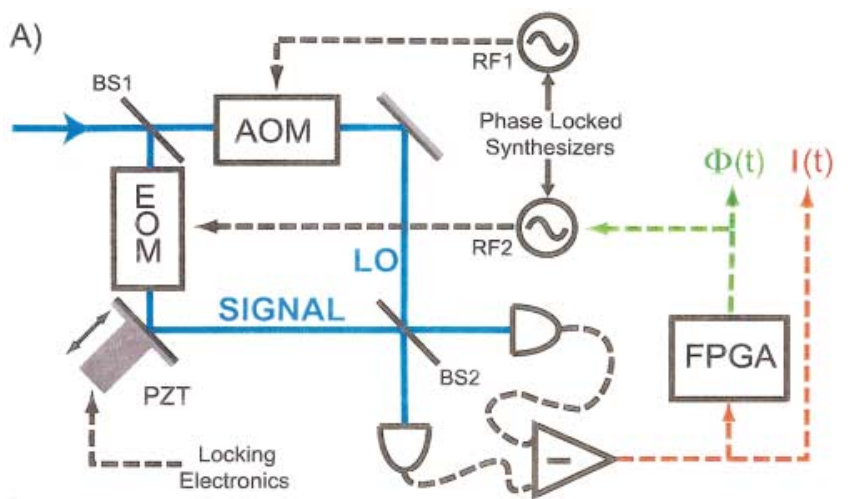

B)

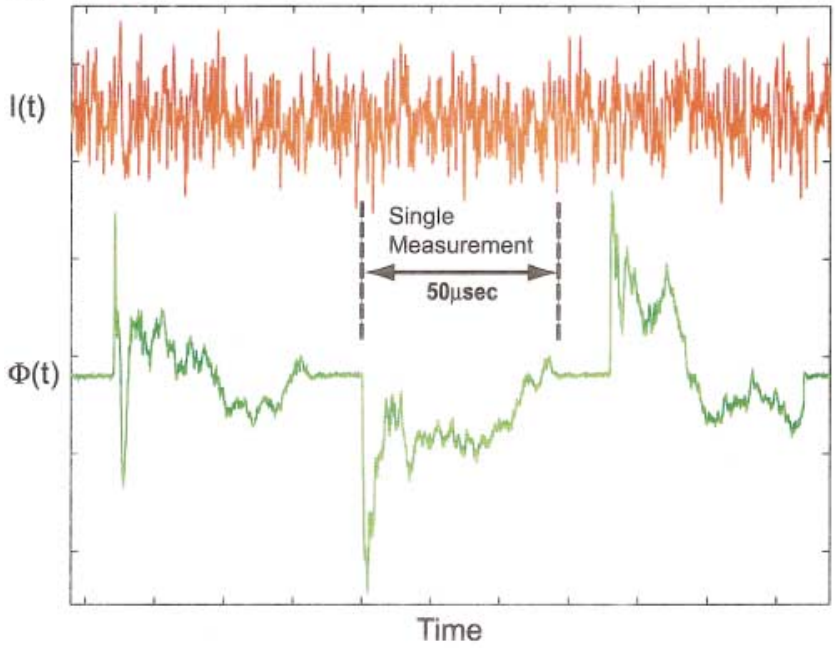

FIG. 2 (color). (a) Apparatus used to perform both adaptive homodyne and heterodyne measurements (see text). Solid lines denote optical paths, and dashed lines denote electrical paths. (b) Photocurrent $I(t)$ (red above), and feedback signal $\Phi(t)$ (green below), for three consecutive adaptive homodyne measurements with $N \approx 8$. The $x$ axis represents time for both signals. The $y$ axis scale indicates the absolute phase shifts made by the feedback signal. The photocurrent is plotted on an arbitrary scale.

[13]. Our feedback and phase-estimation procedures correspond to the "Mark II" scheme of Wiseman and coworkers [11], in which the photocurrent is integrated with time-dependent gain to determine the instantaneous feedback signal: $\Phi(t)-\pi / 2 \propto \int_{0}^{t} d v I(v) / \sqrt{v}$, where $t$ is scaled such that the pulse has duration 1 . The final phase estimate depends on integrals of the photocurrent and feedback signal according to the Mark II scheme. For heterodyne measurements we turn off the feedback to RF2 and detune it from RF1 by $1.8 \mathrm{MHz}$; phase estimates are made by the standard method of I/Q demodulating the photocurrent beat note. For both types of measurement we store the photocurrent $I(t)$ and feedback signal $\Phi(t)$ on a computer for postprocessing.

In Fig. 2(b) we show the photocurrent and feedback signal of three consecutive adaptive phase measurements 
for $N \approx 8$. At the beginning of each measurement, feedback of the photocurrent shot noise causes the relative phase between the signal and LO to vary randomly. As more of the pulse is detected, the information gained is used to drive the signal-LO phase towards an optimal value. Phase estimation variances are established using ensembles of $\approx 150$ consecutive single-shot measurements. Although each optical pulse is the subject of an independent single-shot measurement, we fix the signal phase over the length of each ensemble in order to accurately determine the estimation variance. It is important to note that each data point in Fig. 1(a) corresponds to an average of variance estimates from many ensembles, each with a different (random) signal phase.

Ideally, the performance of a single-shot phase measurement procedure should be independent of the signal phase. In our experiment, this implies that the measurement variances should be independent of the initial relative phase between signal and local oscillator. In Fig. 1(c) we display a polar plot of the adaptive variance (radial) versus initial signal phase (azimuth). The data were taken using signals with mean photon number $\approx 50$. Each data point corresponds to the variance of an ensemble of phase measurements taken at a fixed phase. The double solid lines indicate the $1 \sigma$ scatter of our heterodyne data. It is clear from this data that the performance of the adaptive homodyne scheme is essentially independent of initial phase, and is consistently superior to heterodyne measurement.

The photon number per pulse, $N$, is determined by extracting optical amplitude information from the balanced photocurrent in heterodyne mode. The typical experimental procedure is to fix the signal amplitude, take an ensemble of heterodyne measurements (which yields both heterodyne phase estimates and an estimate of $N$ ), and then take an ensemble of adaptive homodyne measurements. The photon number assigned to the subsequent ensemble of adaptive measurements is $0.95 \mathrm{~N}$, where the relative calibration factor arises from the measured response of the EOM.

In conclusion, we have presented an experimental demonstration of adaptive homodyne phase measurement. For pulses with mean photon number $\sim 10-300$ our measured variances approach closer to the intrinsic phaseuncertainty limit of coherent states than any previously demonstrated technique. These results establish the feasibility of broadband quantum-noise-limited feedback for adaptive quantum measurement [14], quantum feedback control [15], quantum error correction [16], and studies of conditional quantum dynamics $[17,18]$.
This work was supported by the NSF (PHY-9987541, EIA-0086038) and ONR (N00014-00-1-0479).

*Electronic address: armen@caltech.edu

[1] K. Schneider et al., Opt. Express 2, 59 (1998).

[2] See special issue of Phys. Scr. T48 (1993).

[3] U. Leonhardt et al., Phys. Rev. A 51, 84 (1995).

[4] H. M. Wiseman, Phys. Rev. Lett. 75, 4587 (1995).

[5] Note that this precludes the use of techniques such as homodyne tomography, which rely on multiple measurements on an ensemble of identically prepared systems.

[6] C. M. Caves and P. D. Drummond, Rev. Mod. Phys. 66, 481 (1994).

[7] This factor of 2 derives from the fact that heterodyne detection samples both the amplitude and phase-quadrature amplitudes, which are complementary observables.

[8] Y. Yamamoto et al., in Progress in Optics, edited by E. Wolf (North-Holland, Amsterdam, 1990), Vol. 28, p. 150.

[9] Our calibration of the horizontal axis has been adjusted for overall photodetection efficiency $\eta$, which has no bearing on the comparison of adaptive and heterodyne procedures but does affect our placement of curves on the graph; had the measurements been of unit efficiency, the data and theory variances would decrease by a factor of $\eta$. We have independently measured $\eta \approx 0.56$ (detector quantum efficiency $\approx 0.85$, homodyne efficiency $\approx$ $0.66)$. For phase measurements performed on squeezed states $\eta$ would need to be optimized, but its value and affect on the photon-number calibration are not of direct concern in our current work involving coherent states.

[10] For pulses with mean photon number $>10$, an ideal adaptive measurement would lie within $1 \%$ of the uncertainty limit. Accounting for technical noise, the variance of the adaptive measurement could at best lie a factor of 2 below the noise-corrected heterodyne curve.

[11] H. M. Wiseman and R. B. Killip, Phys. Rev. A 57, 2169 (1998).

[12] In principle one can modulate either the signal phase or the LO phase; we do the former to avoid interference between the adaptive homodyne feedback loop and an auxiliary feedback loop used to stabilize the MZI.

[13] J. K. Stockton, M. Armen, and H. Mabuchi, J. Opt. Soc. Am. B (to be published).

[14] D.W. Berry and H. M. Wiseman, Phys. Rev. A 63, 013813 (2001).

[15] A. C. Doherty et al., Phys. Rev. A 62, 012105 (2000).

[16] C. Ahn, A. C. Doherty, and A. Landahl, Phys. Rev. A, 65, 042301 (2002).

[17] W. P. Smith et al., Phys. Rev. Lett. 89, 133601 (2002).

[18] H. Mabuchi and H. M. Wiseman, Phys. Rev. Lett. 81, 4620 (1998). 